\title{
GutGame Digital: Um Jogo de Apoio ao Ensino de Fisiologia Humana
}

\author{
Washington P. Batista ${ }^{1}$, Lenington C. Rios ${ }^{1}$, Victor T. Sarinho ${ }^{1}$, \\ Érica M. Granjeiro ${ }^{1}$, Rafael P. de Campos $^{2}$, Natascha M. Medeiros ${ }^{2}$, \\ Victória P. Viero ${ }^{3}$, Lucila L. P. Gutierrez ${ }^{3}$ \\ ${ }^{1}$ Universidade Estadual de Feira de Santana - UEFS \\ Feira de Santana - Bahia - Brasil \\ \{wstroks, lenitorios\}@gmail.com, \{vsarinho, ericag\}@uefs.br \\ ${ }^{2}$ Universidade Federal do Rio Grande do Sul - UFRGS \\ Porto Alegre - Rio Grande do Sul - Brasil \\ rafa.paschoal@yahoo.com.br, natamoonmedegmail.com \\ ${ }^{3}$ Universidade Federal de Ciências da Saúde de Porto Alegre - UFCSPA \\ Porto Alegre - Rio Grande do Sul - Brasil \\ vierovictoria@gmail.com, lucilagutierrez@yahoo.com.br
}

\begin{abstract}
Nowadays, students face difficulties in memorizing structures of $\mathrm{Hu}$ man Physiology (HP) within a teaching that induces them to think about functional processes in a fragmented way. Proposing a differentiated and motivating way to study HP, this article presents GutGame Digital, a digital board-style game that seeks to support the teaching of HP. It is a game that reproduces in the digital environment questions and operating logic applied in the physical version of the same, which was used in the construction of differentiated learning dynamics for higher education in HP in the classroom.
\end{abstract}

Resumo. Atualmente, estudantes enfrentam dificuldade de memorização de estruturas de Fisiologia Humana (FH) dentro de um ensino que induz a pensar nos processos funcionais de maneira fragmentada. Propondo uma forma diferenciada e motivadora para o estudo de FH, este artigo apresenta o GutGame Digital, um jogo digital estilo tabuleiro que busca dar apoio ao ensino de FH. Trata-se de um jogo que reproduz no meio digital questões e lógica de funcionamento aplicadas na versão física do mesmo, o qual foi utilizado na construção de dinâmicas de aprendizagem diferenciadas para o ensino superior de FH em sala de aula.

\section{Introdução}

O ensino da Fisiologia Humana (FH) "costuma ser dividido em sistemas biológicos" cujo objetivo é facilitar sua aprendizagem, porém, seguindo uma abordagem que deturpa a integração do organismo "levando o aluno a pensar nos processos funcionais de maneira fragmentada", gerando como resultado dificuldades no processo de aprendizado e entendimento a respeito do assunto [de Araújo Alves et al. 2016] [Vanzela et al. 2013]. 
O ensino das áreas biológicas tem por objetivo "estimular a construção de um aprendizado significativo que supere apenas a memorização de nomes, regras e leis" [Merçon 2015]. Para isso, o aluno deve receber um ensino que seja capaz de fazêlo: compreender interações entre organismos e ambientes relacionando conhecimentos científicos, aspectos culturais e características individuais; identificar padrões em fenômenos e processos vitais dos organismos; e interpretar modelos e experimentos para explicar os fenômenos ou processos biológicos em qualquer nível de organização dos sistemas biológicos [de Araújo Alves et al. 2016].

A compreensão e a aprendizagem de conhecimentos "são facilitadas quando tomam a forma aparente de atividade lúdica" [Campos et al. 2003], visto que os estudantes mostram entusiasmo quando sua aprendizagem é de uma forma mais intuitiva, interativa e divertida, podendo resultar em um aprendizado mais significativo. Neste sentido, jogos digitais vem ganhando espaço como uma ferramenta primordial da aprendizagem, motivando o aluno e desenvolvendo níveis diferentes de experiência pessoal, de modo a auxiliar a construção de novas descobertas, o desenvolvimento de personalidade, entre outros aspectos positivos [Oliveira Neto and Ribeiro 2012].

Este artigo apresenta o GutGame Digital, um jogo digital estilo tabuleiro que busca dar apoio ao ensino de FH. Trata-se de um jogo que reproduz no meio digital questões e lógica de funcionamento aplicadas na versão física do mesmo, o qual foi utilizado na construção de dinâmicas de aprendizagem diferenciadas para o ensino de $\mathrm{FH}$ em turmas de ensino universitário na área de saúde.

\section{Trabalhos Relacionados}

Diversos trabalhos têm sido desenvolvido nos últimos anos com o objetivo de unir ensino e diversão através de jogos para a área de biologia [Borges et al. 2016]. Como exemplo, o jogo de tabuleiro "Evolução: A luta pela Sobrevivência" trata da evolução de animais vertebrados [Campos et al. 2003], onde os jogadores têm que se movimentar em um tabuleiro ao longo do tempo geológico, passando por todas as evoluções e vivenciando o que aconteceu com cada grupo de vertebrados. Já o jogo "Heredograma sem Mistério" trata de conceitos de genética [Campos et al. 2003], onde os participantes devem elaborar um heredograma sobre cor de olhos, sistema sangüíneo ABO ou daltonismo, de acordo com as instruções do respectivo cartão de caso, que deve ser escolhido pelo professor. $\mathrm{O}$ jogo de cartas "Baralho didático: temas de biologia para ensino médio" abrange temas como vírus, bactérias, algas, protozoários e fungos [Fernandes et al. 2014], e o grupo que primeiro formar a sequência correta de algum dos temas propostos, ou seja, fizer corretamente as correlações conceito/imagem dos elementos do jogo ganha a partida. Para finalizar, o jogo Body Digital [Borges et al. 2016] apresenta a aplicação de mecânicas e dinâmicas do jogo War para a conquista de órgãos do corpo humano de uma maneira colaborativa e ativa.

\section{Metodologia}

O jogo Gutgame Digital é fruto da parceria de professores da UEFS e da UFCSPA com o objetivo de transformar o jogo GutGame [de Campos et al. 2020] já aplicado em sala de aula em um jogo digital de fácil acesso aos alunos. Para tal, fez-se um estudo de como o jogo foi aplicado em sala de aula, de modo a produzir uma adaptação viável e adequada para o meio digital. 
Sendo recolhida as informações necessárias a partir deste estudo, percebeu-se que o jogo contavam com perguntas de 4 alternativas para cada parte do orgão humano. Assim, quando o jogador acertava/errava uma pergunta, ele poderia avançar/permanecer conforme sua localização no tabuleiro do jogo. Para fins de ilustração, a Figura 1 apresenta um exemplo de material físico do jogo refatorado para o meio digital, onde a letra A corresponde a imagem original do jogo e a letra $\mathrm{B}$ corresponde a imagem que foi refatorada e digitalizada para o jogo.

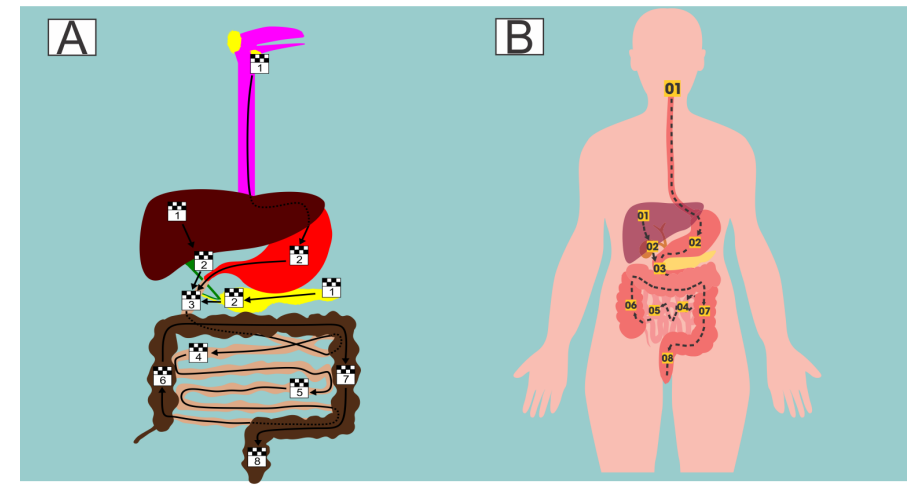

Figura 1. Refatoração das imagens físicas do GutGame para o meio digital.

Perante essa estrutura, seguiu-se com a produção de um jogo estilo tabuleiro para o meio digital, onde o jogador tem que passar por "casas" ou fases que correspondem aos órgãos do corpo humano para vencer o jogo. Como o intuito do GutGame Digital é avaliar conhecimentos de FH, foi mantida a estrutura de perguntas e respostas do jogo original, por um intermédio de um Quiz de cada orgão, sendo que 5 perguntas são apresentadas para cada casa. Diante desse contexto, para o usuário seguir o fluxo e chegar a casa final do GutGame, é necessário acertar no mínimo 3 questões e assim conseguir avançar de casa.

Para a etapa de construção e elaboração do jogo, fez-se necessária a criação de um design para as interfaces do jogo e a sua implementação para os ambientes web e mobile. Para tal, utilizou-se a ferramenta Godot, um motor de construção de jogos open source que permite um desenvolvimento ágil atrelado com a complexidade das interfaces e animações presentes no jogo (Figura 2).

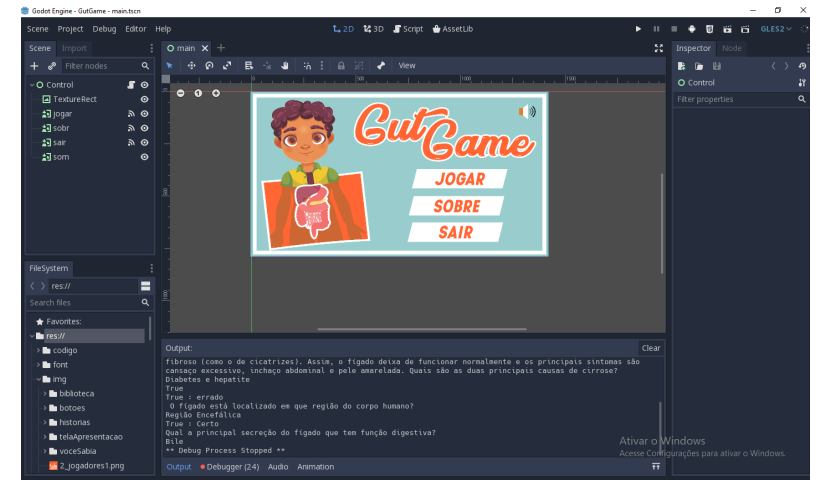

Figura 2. Telas principais do GutGame Digital na ferramenta Godot.

Como a mecânica principal do GutGame Digital é baseada em um Quiz, tem-se 
que a ordenação das perguntas se faz de forma aleatória, para que as respostas do jogador não se tornem automáticas caso ele perca ou jogue o jogo uma outra vez. Vale ressaltar que cada casa tem um total de 15 perguntas, criado assim um sistema rotativo para que não ocorra sempre as mesmas perguntas para o jogador. Uma das maneiras apresentadas para orientar o jogador se ele acertou a pergunta ou não é através de expressões faciais, ou seja, quando o usuário acerta o jogo mostra um personagem com a expressão feliz e quando erra uma expressão triste.

Por fim, o GutGame em sua versão digital apresenta dois módulos de jogos, sendo um single player, no qual o jogador escolhe a casa que deseja iniciar, seja ela "Fígado ou Boca", e o módulo multiplayer, que pode ser jogado até com "4 Jogadores". Com relação ao fluxo de execução de cada jogador no tabuleiro virtual do jogo, tem-se que as informações salvas para cada jogador, de modo a garantir um fluxo adaptável a qualquer caminho que o jogador percorra, são: número de acertos no Quiz para a casa corrente; avanços de "casas" na partida; número de acertos geral no Quiz; e jogador corrente da rodada no caso do multiplayer. Vale ressaltar que o multiplayer é local, onde os jogadores jogam na mesma tela de computador, sendo determinado quando é a vez de cada um pelo jogo.

\section{Resultados e Discussões}

O jogo produzido mostra uma interface de fácil utilização para o usuário, oferecendo menus e ações de forma simples e direta. A Figura 3 mostra quatro telas da aplicação, sendo a Figura 3.A o menu principal contendo as opções "Jogar", "Sobre" e "Sair". A Figura 3.B exibe as opções para os modos single player e multiplayer do jogo. Ao escolher o modo single player, o usuário é direcionado para a tela da Figura 3.C na qual deverá escolher o local onde incia o jogo, sendo "Boca e Esôfago" ou "Fígado". Ao escolher a opção multiplayer, será redirecionado para a tela Figura 3.D na qual deverá escolher quantos jogadores irão utilizar a aplicação (2, 3 ou 4 jogadores).

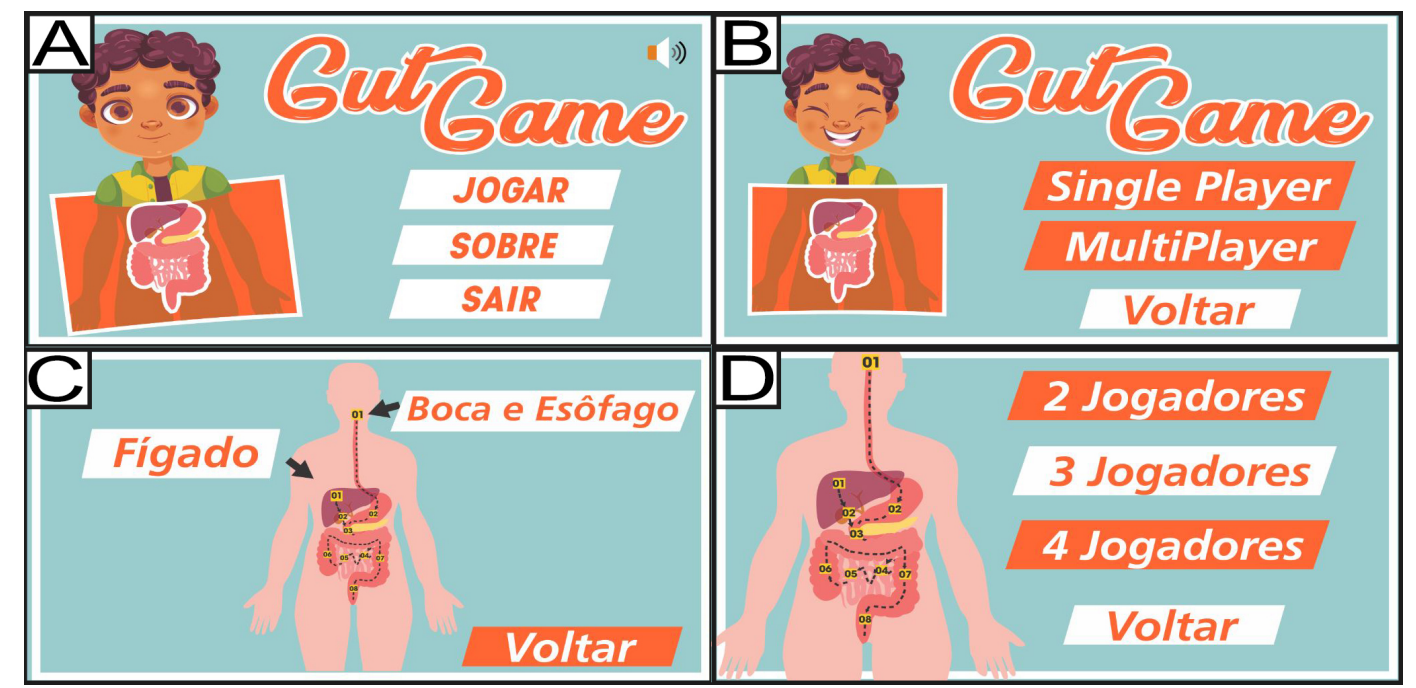

Figura 3. Telas principais do GutGame.

Já na Figura 4.A, é apresentado um feedback com relação a se os jogadores conseguiram ou não avançar de "casas", e em sequência, na 4.B, é indicado pelas "setas" onde 
o jogador está localizado no tabuleiro. No modo multiplayer os locais de cada jogador são apresentados no tabulerio de forma simultânea, como pode ser visto no exemplo de "2 jogadores" na Figura 4.C. Por fim, o jogador que conseguir passar por todas as casas primeiro é o vencedor do jogo, o qual é ilustrado na Figura 4.D indicando o jogador vencedor e dando sua parabenização pela vitória no mesmo.

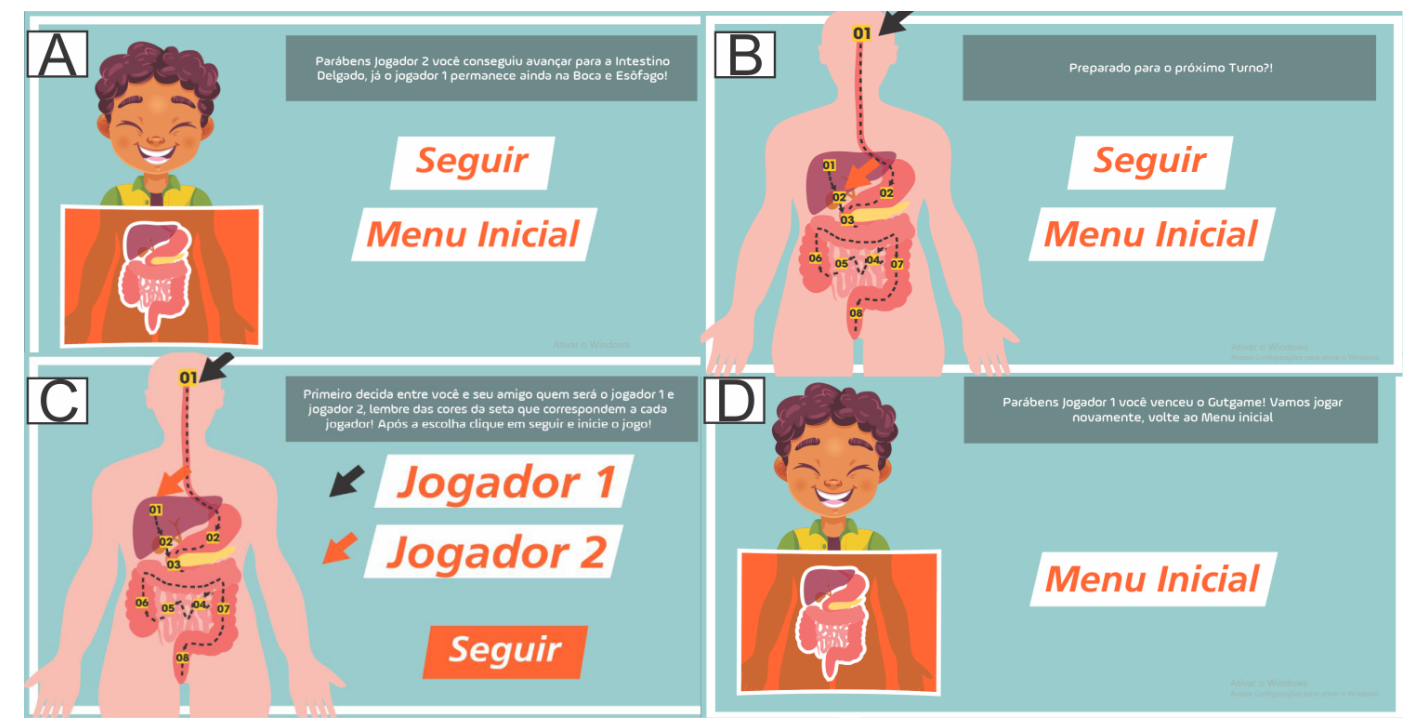

Figura 4. Telas de informações do jogo.

Com relação aos feedbacks para os jogadores, elaborou-se uma animação para as respostas aos quizzes no jogo, conforme ilustrado na Figura 5. Nela são apresentadas 2 imagens, onde quando o jogador acerta a pergunta ele recebe um expressão de felicidade e caso contrário é apresentado a expressão de tristeza. O mesmo caso ocorre para o módulo multiplayer, com a diferenciação que estará contido na tela, de quem é a vez, como por exemplo "Jogador 1".

\section{Conclusões e Trabalhos Futuros}

Este artigo apresentou o desenvolvimento do GutGame Digital, um jogo educativo que tem o intuito de transmitir informações sobre FH através de uma dinâmica de tabuleiro com avanços a partir de perguntas e respostas apresentadas aos jogadores. Trata-se de uma versão digital de um jogo já aplicado em sala de aula, projetado em uma versão $w e b$ com intuito de deixar o jogo disponível on-line (http: / / gut game . herokuapp.com/) e minimizar a presença de um "mestre" para administrar as perguntas, e consequentemente o feedback necessário nas respostas.

Como trabalhos futuros, pretende-se realizar a aplicação do mesmo em sala de aula, bem como o compartilhamento opcional de conquistas do mesmo em mídias sociais para fins de viralização futura do jogo. Outro ponto futuro a ser trabalhado é na melhoria da mecânica do jogo, como a construção de mini-games ao invés da estrutura de um Quiz. Como exemplo, pode-se apresentar uma situação em que o personagem tenha "gastriste" e o jogador deva apenas clicar em comidas saudáveis para não agravar a situação do personagem, e consequentemente avançar de "casa" durante uma partida. 


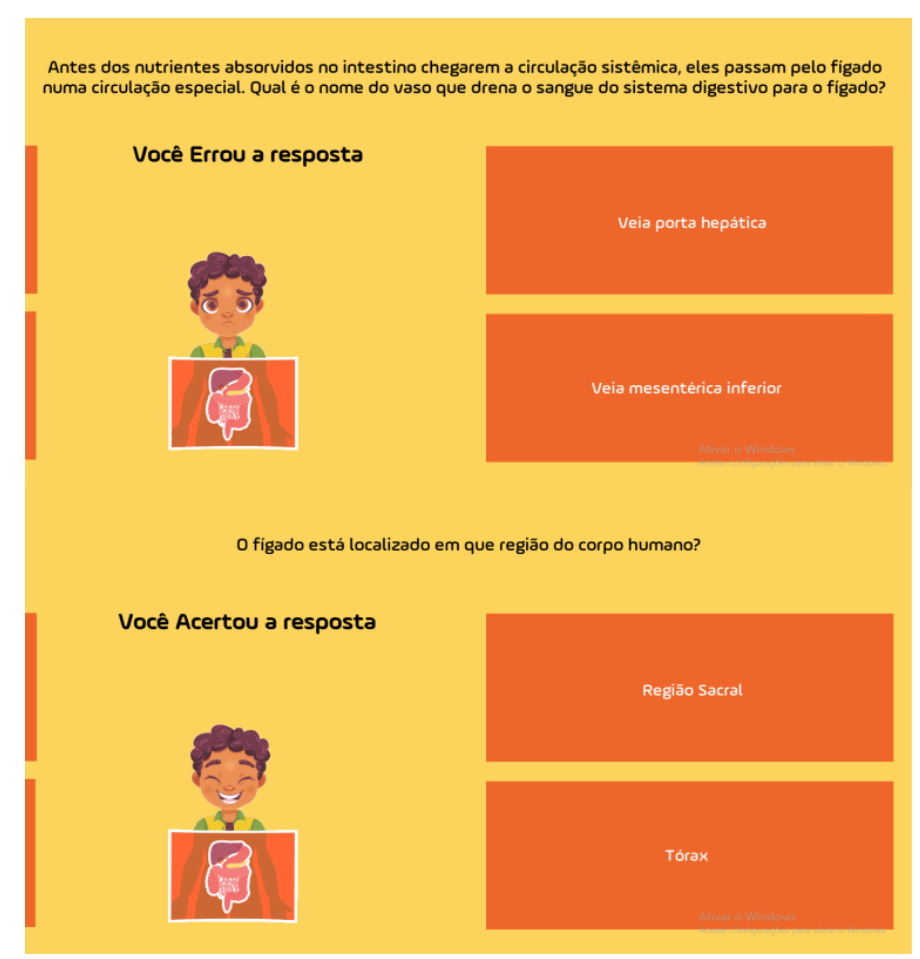

Figura 5. Exemplo de Quiz com animação apresentado no jogo.

\section{Referências}

Borges, G. A., Lima, C. O. C., Granjeiro, E. A., Sarinho, V. T., and Bittencourt, R. A. (2016). Body: Um jogo digital educacional de tabuleiro na area de fisiologia humana. In Proceedings of SBGames, 2016. XV SBGames, pages 412-420. SBC.

Campos, L. M. L., Bortoloto, T. M., and Felício, A. K. C. (2003). A produção de jogos didáticos para o ensino de ciências e biologia: uma proposta para favorecer a aprendizagem. Caderno dos núcleos de Ensino, 3548.

de Araújo Alves, T., de Souza Falcão, L., Souza, A. T., do Amaral, T. S., de Lima, S. P., and Carvalho, T. B. (2016). Fisio card game: um jogo didático para o ensino da fisiologia na educação básica. Revista de Ensino de Bioquímica, 14(1):99-120.

de Campos, R. P., Viero, V. P., Medeiros, N. M., Marcondes, F. K., Montrezor, L. H., Porawski, M., and Gutierrez, L. L. P. (2020). The "gut game": An active methodology to teach digestive physiology. Advances in Physiology Education.

Fernandes, S. M. A., Mavignier, R. D., Silva, R. D. S., Silva, F., and Dantas, S. (2014). Baralho didático: Temas de biologia para ensino médio. Revista da SBEnBio,(7).

Merçon, F. (2015). Os objetivos das ciências naturais no ensino médio. Revista Eletrônica do Vestibular.[periódicos na internet].

Oliveira Neto, A. A. d. and Ribeiro, S. A. B. (2012). Um modelo de role-playing game (rpg) para o ensino dos processos da digestão.

Vanzela, E. C., Balbo, S. L., and Della Justina, L. A. (2013). A integração dos sistemas fisiológicos e sua compreensão por alunos do nível médio. Arquivos do Museu Dinâmico Interdisciplinar, 11(3):12-19. 